\title{
Abdominal Aortic Aneurysms and Coronary Artery Disease in a Small Country with High Cardiovascular Burden
}

\author{
Hassan Al-Thani ${ }^{1,2}$ and Ayman El-Menyar ${ }^{3,4,5}$ \\ ${ }^{1}$ Vascular Surgery, Hamad General Hospital, P.O. Box 3050, Doha, Qatar \\ ${ }^{2}$ General Surgery, Hamad General Hospital, Doha, Qatar \\ ${ }^{3}$ Clinical Research, Hamad General Hospital, HMC, P.O. Box 3050, Doha, Qatar \\ ${ }^{4}$ Clinical Medicine, Weill Cornell Medical School, P.O. Box 24144, Doha, Qatar \\ ${ }^{5}$ Cardiology Unit, Internal Medicine, Ahmed Maher Teaching Hospital, Cairo, Egypt \\ Correspondence should be addressed to Ayman El-Menyar; aymanco65@yahoo.com
}

Received 24 November 2013; Accepted 13 January 2014; Published 20 February 2014

Academic Editors: A. Becker, J. A. F. Ramires, and A. Stephanou

Copyright (c) $2014 \mathrm{H}$. Al-Thani and A. El-Menyar. This is an open access article distributed under the Creative Commons Attribution License, which permits unrestricted use, distribution, and reproduction in any medium, provided the original work is properly cited.

\begin{abstract}
We aimed to evaluate the frequency, clinical profiles and outcomes of abdominal aortic aneurysms (AAA), and their association with coronary artery disease (CAD) in a small country with high cardiovascular burden. Methods. Data were collected for all adult patients who underwent abdominal computed tomography scans at Hamad General Hospital in Qatar between 2004 and 2008. Results. Out of 13,115 screened patients for various reasons, 61 patients $(0.5 \%)$ had abdominal aneurysms. The majority of AAA patients were male (82\%) with a mean age of $67 \pm 12$ years. The incidence of AAA substantially increased with age reaching up to $5 \%$ in patients $>80$ yrs. Hypertension was the most prevalent risk factor for AAA followed by smoking, dyslipidemia, renal impairment, and diabetes mellitus. CAD and peripheral arterial disease (PAD) were observed in $36 \%$ and $13 \%$ of AAA patients, respectively. There were no significant correlations between CAD or PAD and site and size of AAA. Conclusion. This is the largest study in our region that describes the epidemiology of AAA with concomitant CAD. As the mortality rate is quite high in this high risk population, routine screening for AAA in CAD patients and vice versa needs further studies for proper risk stratification.
\end{abstract}

\section{Introduction}

Screening programs for abdominal aortic aneurysm (AAA) are lacking in the developing countries. Global data reported that the prevalence of AAA varies in men $(1.3 \%-8.9 \%)$ and women $(11 \%-2.2 \%)$ [1].

The rising incidence of AAA is related mainly to the increase in age, physician awareness with clinical high index of suspicion, and the use of advanced diagnostic modalities. As AAA is a silent process, it may present only with aneurysmal rupture in most of cases. It was believed for a long time that atherosclerosis is the main pathogenesis of AAA. However, this speculation has raised a question that whether atherosclerosis is a "bystander" condition or an active factor for the initiation or acceleration of AAA $[1,2]$.
In a systematic review, Elkalioubie et al. evaluated 17 published studies between 1991 and 2010 [1]. The authors found that the frequency of AAA among coronary artery disease patients ranged between $0.48 \%$ and $18.2 \%$ [1]. As advanced age is a potential risk factor for both coronary artery disease and AAA, five studies specifically recruited patients who were above 60 years. Salem et al. [3] reported a lower prevalence of AAA among men of Asian origin (China and Iran), indicating that certain ethnic groups experience a disproportionately smaller burden of AAA. Notably, the prevalence of AAA in coronary artery disease patients was comparable to that reported in AAA screening studies among patients with different vascular bed affection such as peripheral vascular disease (PAD) $(7 \%-17 \%)$ and cerebrovascular disease $(8.4 \%-20.2 \%)[1,4,5]$. Herein, we aim to evaluate the frequency, clinical profiles and outcomes of AAA, and 
TABLE 1: Clinical presentation and outcome of AAA.

\begin{tabular}{lcc}
\hline & Number of patients & $\%$ \\
\hline Total AA & 61 & $0.5 \%$ \\
Age, median & \multicolumn{2}{c}{$69(26-88)$} \\
Age $\geq 65$ yrs & 41 & $67 \%$ \\
Infrarenal AAA & 41 & 67 \\
Thoracoabdominal & 14 & 23 \\
AAA rupture & 5 & 8 \\
Died & 16 & 33 \\
Male & 50 & 82 \\
Diabetics & 25 & 41 \\
Hypertension & 40 & 66 \\
Dyslipidemia & 31 & 51 \\
Smoking & 37 & $60 \%$ \\
Coronary artery disease & 22 & 36 \\
Size of AAA & Mean $5.3 \pm 2.5$, median $4.8(3-14)$ \\
Size $\geq 5.5 \mathrm{~cm}$ & 22 & $41 \%$ \\
Size $\geq 7$ cm & 14 & $26 \%$ \\
\hline
\end{tabular}

its coronary artery disease association in Qatar. Qatar is a small rapidly developing country in the Middle East that is characterized with high prevalence of cardiovascular risk burden $[6,7]$.

\section{Methods}

Data were collected from March 2004 to March 2008 for all adult patients who underwent abdominal computed tomography scans at Hamad General Hospital in Qatar. All adult patients who underwent abdominal CT scans for any reason were included in the study and those with diagnosed abdominal aneurysms were followed up for 3 years for major events (AAA rupture and mortality). AAA was defined as an abnormal dilatation of the abdominal aorta with a maximal diameter of $\geq 3 \mathrm{~cm}$. CAD was defined as history of documented coronary events, history of percutaneous or surgical coronary intervention or currently on anti-ischemic medications. PAD was defined as intermittent claudication, vascular lab diagnosis, or lower-limb arterial revascularization [7]. Data were reported in percentage, mean $\pm S D$, and median and range, when applicable. Data analysis was carried out using the Statistical Package for Social Sciences version 18 (SPSS Inc. USA).

\section{Results}

Out of 13,115 screened patients for various reasons, 61 patients $(0.5 \%)$ were identified to have abdominal aneurysms. The majority of AAA patients were male $(82 \%)$ with a mean age of $67 \pm 12$ years (Table 1). The incidence of AAA substantially increased with age reaching up to $5 \%$ in elderly patients (>80 years). The main location of AAA was infrarenal (67\%), followed by thoracoabdominal (23\%). The mean AAA diameter was $5.3 \pm 2.5 \mathrm{~cm}$ with a range from 3 to $14 \mathrm{~cm}$. AAA

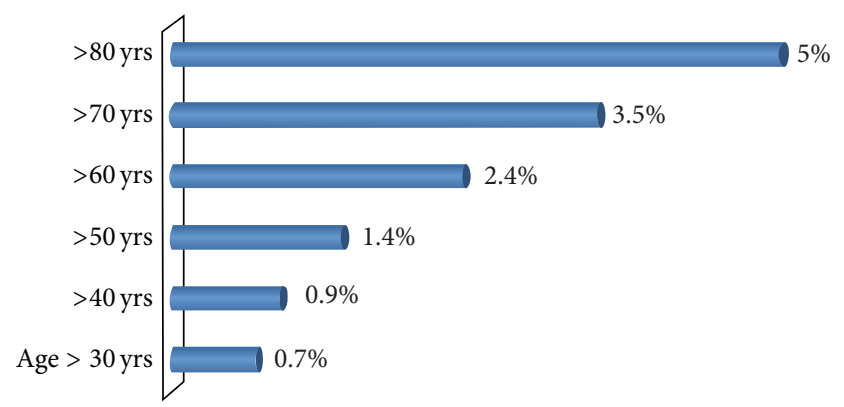

FIGURE 1: Abdominal aortic aneurysm in different age groups.

TABLE 2: AAA with history of coronary artery disease.

\begin{tabular}{lc}
\hline Age in yrs, median (range) & $72(44-82)$ \\
Male gender \% & 91 \\
Diabetes mellitus \% & 54.5 \\
Hypertension \% & 86.5 \\
Dyslipidemia \% & 82 \\
Peripheral arterial disease \% & 23 \\
Age $\geq 60$ yrs & $91 \%$ \\
AAA location & \\
$\quad$ Infrarenal & $68 \%$ \\
$\quad$ Thoracoabdominal & $18 \%$ \\
AAA size (cm) & \\
$\quad \geq 5.5$ & $30 \%$ \\
$\quad \geq 7$ & $20 \%$ \\
Ruptured AAA & $4.5 \%$ \\
Died & $47 \%$ \\
\hline
\end{tabular}

of diameter $\geq 5.5$ and $\geq 7 \mathrm{~cm}$ comprised $41 \%$ and $26 \%$ of cases, respectively. Figure 1 shows the AAA in different age groups.

The rate of AAA rupture was $8 \%$, with an overall mortality of $33 \%$. Among ruptured AAA, all patients were above 60 years old; $80 \%$ had AAA diameter of $\geq 5.5 \mathrm{~cm}$ and $60 \%$ of them died. Moreover, $80 \%$ of AAA located infrarenally.

Cardiovascular risk factors were prevalent among AAA patients in terms of hypertension (66\%), smoking (60\%), dyslipidemia (51\%), renal impairment (46\%), and diabetes mellitus $(41 \%)$. CAD was prevalent in more than one-third of cases $(36 \%)$, whereas PAD was observed in $13 \%$ of AAA patients. There were no significant correlations between CAD or PAD and site and size of AAA.

Figure 2 shows the rates of AAA rupture and deaths in different AAA size groups.

Table 2 shows the clinical profile of patients with AAA who had concomitant CAD. Patients with coexistent CAD were older (median age of 72 years); $91 \%$ of them were males and had high prevalence of cardiovascular risk factors as hypertension (86\%), dyslipidemia (82\%), and diabetes mellitus (54.5\%). The mortality rate was $47 \%$ in patients with combined AAA and CAD, while AAA patients without CAD had $26 \%$ mortality rate. 
TABLE 3: Factors associated with increased risk of developing an AAA.

Old age

Gender

Men develop AAA 4-5 times more often than women

Ethnicity

White people develop AAA more frequently than other ethnicities

Vascular bed affection

People with CAD and PAD are more likely to develop AAA than those who are otherwise healthy

Family history

A family history of AAA increases the risk of developing AAA

The risk of developing an AAA among brothers of a patient with a known AAA who are $>60$ years old is as high as $18 \%$

Cardiovascular risk factors

(i) Smoking: the risk is directly related to number of years smoking

(ii) Diabetes mellitus: there is a negative association with diabetes mellitus and AAA

(iii) Hypertension is a poor predictor for AAA development but important risk factor for expansion and rupture

(iv) Lipid: there is no and weak correlation between risk for AAA and high serum triglyceride and cholesterol, respectively

Recommendations for AAA screening

Men of age 65-75 who have ever smoked should be screened one time for AAA with abdominal ultrasound

Men $>75$ are unlikely to benefit from screening

Men age $\geq 60$ who have a sibling or parent with an AAA should have a physical examination and abdominal ultrasound

There is no recommendation for general screening for AAA in women

(i) Women who have an increased risk for AAA (those who smoke have a family history of -AAA, or other risk factors) should be put into consideration

(ii) The risk of rupture in women is higher than in men, and so some data are in favor of one-time screening for women with risk factors

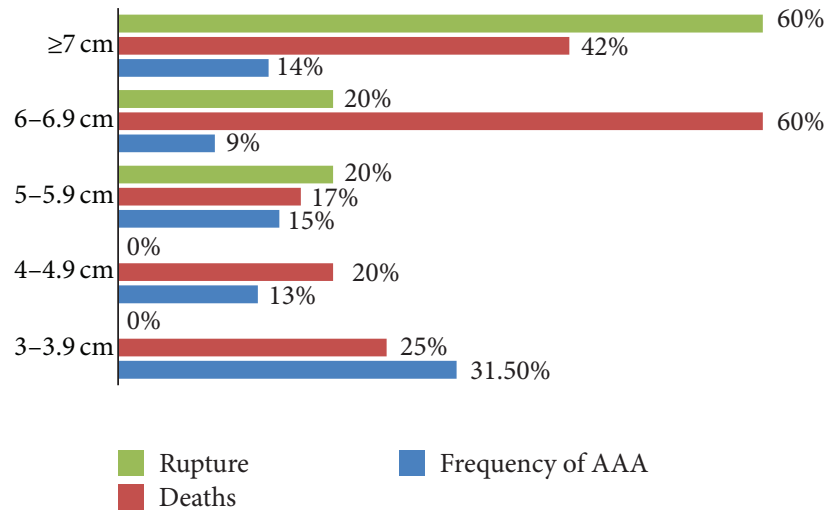

Figure 2: Rates of AAA rupture and deaths in different AAA size groups.

\section{Discussion}

This is the largest study that describes the frequency of incidental AAA and concomitant CAD in the Arab Gulf Region. In our data, incidental abdominal aneurysms were detected in $0.5 \%$ of screened patients, of which the majority $(67 \%)$ was $\geq 65$ years old. The cardiovascular risk factors were prevalent among AAA patients in our study. Two-thirds of AAA patients were hypertensive, $60 \%$ were smokers, about half had dyslipidemia, and $41 \%$ had diabetes mellitus. History of CAD and PAD was observed in $36 \%$ and $13 \%$ of AAA patients, respectively. Among CAD patients, atherosclerosis risk factors such as age, smoking, and existence of other vascular bed affection showed an association with AAA [1]. This association addresses the need for screening for AAA in high risk population. Our analysis showed that the mortality was almost 2-fold greater in AAA patients if the history of CAD is present. Table 3 summarizes the factors associated with increased risk of developing AAA and the recommendations for routine screening for AAA $[8,9]$.

It has been shown that not all patients with atherosclerotic disease develop an AAA. Few studies reported that many AAA patients lack concomitant atherosclerotic arterial disease [1]. Moreover, previous data did not correlate the existence or severity of each disease in the presence of the other one. Interestingly, this doubtful association presents in spite of the fact that AAA and atherosclerosis have several risk factors in common, such as male gender, increased age, smoking, and hypertension [10]. Some investigators believe that it would be useful to assess the relationship between the extent of coronary lesion and infrarenal aortic diameter and/or risk of AAA rupture [1]. On the other hand, some population-based studies showed that AAA is independently associated with preexisting CAD [11].

However, the high incidence of $\mathrm{CAD}$ among AAA patients was reported with an impact on the short-term 
survival post-AAA repair [12]. Studies that evaluated the coronary artery status prior to aortic surgery found a concomitant CAD prevalence from $31 \%$ to $90 \%$ [1, 11-13].

The retrospective nature of the current study is a considerable limitation. The breakdown and severity of CAD based on coronary angiographic features are lacking.

\section{Conclusion}

This is the largest study in our region that describes the epidemiology of abdominal aneurysm with concomitant CAD. The high prevalence of CAD does not express site, size, or severity of AAA so far. As the mortality rate is quite high in this high risk population, routine screening for AAA in CAD patients and vice versa needs further studies for proper risk stratification.

\section{Disclosure}

All the authors read and approved the paper with no conflict of interests and no financial issue to disclose. The Medical Research Center at HMC, Qatar, approved the study with IRB\#12112/12. This study was presented in part at 10th International Congress on Coronary Artery Disease in Florence, Italy, October 13-16, 2013.

\section{Conflict of Interests}

The authors declare that there is no conflict of interests regarding the publication of this paper.

\section{Acknowledgment}

The authors thank the vascular surgery team for their cooperation.

\section{References}

[1] A. Elkalioubie, B. Jude, and A. Dupont, "Abdominal aortic aneurysm in patients with coronary artery disease: a review article," in Diagnosis and Treatment of Abdominal and Thoracic Aortic Aneurysms Including the Ascending Aorta and the Aortic Arch, R. Grundmann, Ed., 2011.

[2] A. F. Trollope and J. Golledge, "Angiopoietins, abdominal aortic aneurysm and atherosclerosis," Atherosclerosis, vol. 214, no. 2, pp. 237-243, 2011.

[3] M. K. Salem, H. S. Rayt, G. Hussey et al., "Should asian men be included in abdominal aortic aneurysm screening programmes?" European Journal of Vascular and Endovascular Surgery, vol. 38, no. 6, pp. 748-749, 2009.

[4] H. A. J. M. Kurvers, Y. van der Graaf, J. D. Blankensteijn, F. L. J. Visseren, and B. C. Eikelboom, "Screening for asymptomatic internal carotid artery stenosis and aneurysm of the abdominal aorta: comparing the yield between patients with manifest atherosclerosis and patients with risk factors for atherosclerosis only," Journal of Vascular Surgery, vol. 37, no. 6, pp. 1226-1233, 2003.

[5] A. Palazzuoli, M. Gallotta, G. Guerrieri et al., "Prevalence of risk factors, coronary and systemic atherosclerosis in abdominal aortic aneurysm: comparison with high cardiovascular risk population," Vascular Health and Risk Management, vol. 4, no. 4, pp. 877-883, 2008.

[6] A. El-Menyar, M. Zubaid, A. Shehab et al., "Prevalence and impact of cardiovascular risk factors among patients presenting with acute coronary syndrome in the middle east," Clinical Cardiology, vol. 34, no. 1, pp. 51-58, 2011.

[7] H. Al-Thani, A. El-Menyar, A. Shabana et al., "Incidental abdominal aneurysms: a retrospective study of 13115 patients who underwent a computed tomography scan," Angiology, 2013.

[8] U.S. Preventive Services Task Force, "Screening for abdominal aortic aneurysm: recommendation statement," Annals of Internal Medicine, vol. 142, no. 3, pp. 198-202, 2005.

[9] J.-P. Van Kuijk, W.-J. Flu, M. Dunckelgrun, J. J. Bax, and D. Poldermans, "Coronary artery disease in patients with abdominal aortic aneurysm: a review article," Journal of Cardiovascular Surgery, vol. 50, no. 1, pp. 93-107, 2009.

[10] S. H. Forsdahl, K. Singh, S. Solberg, and B. K. Jacobsen, "Risk factors for abdominal aortic aneurysms: a 7-year prospective study: the tromsø study, 1994-2001," Circulation, vol. 119, no. 16, pp. 2202-2208, 2009.

[11] K. C. Kent, R. M. Zwolak, N. N. Egorova et al., "Analysis of risk factors for abdominal aortic aneurysm in a cohort of more than 3 million individuals," Journal of Vascular Surgery, vol. 52, no. 3, pp. 539-548, 2010.

[12] V. Falk, T. Walther, and F. W. Mohr, "Abdominal aortic aneurysm repair during cardiopulmonary bypass: rationale for a combined approach," Cardiovascular Surgery, vol. 5, no. 3, pp. 271-278, 1997.

[13] R. Sukhija, W. S. Aronow, K. Yalamanchili, N. Sinha, and S. Babu, "Prevalence of coronary artery disease, lower extremity peripheral arterial disease, and cerebrovascular disease in 110 men with an abdominal aortic aneurysm," American Journal of Cardiology, vol. 94, no. 10, pp. 1358-1359, 2004. 


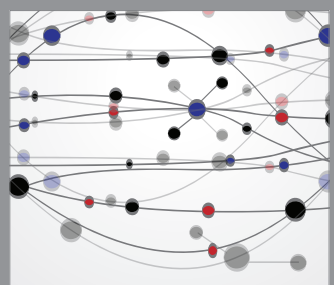

The Scientific World Journal
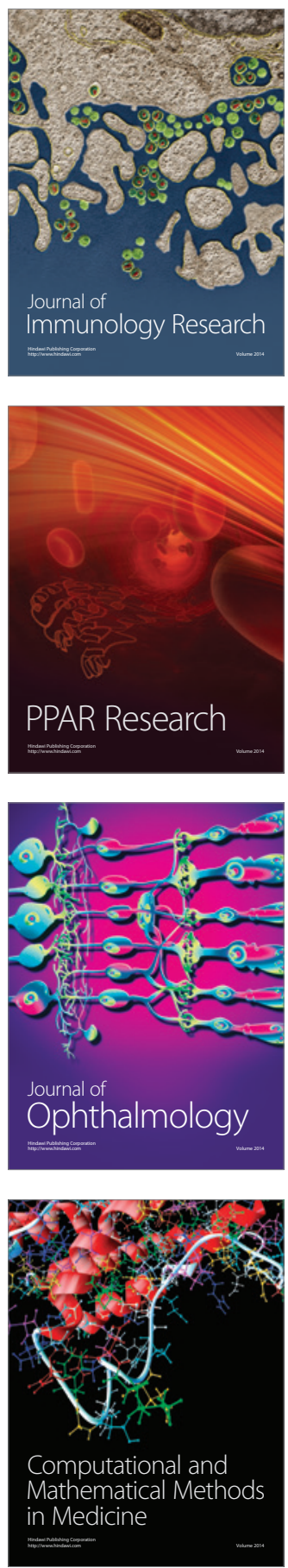

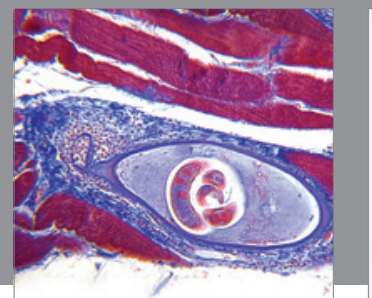

Gastroenterology

Research and Practice
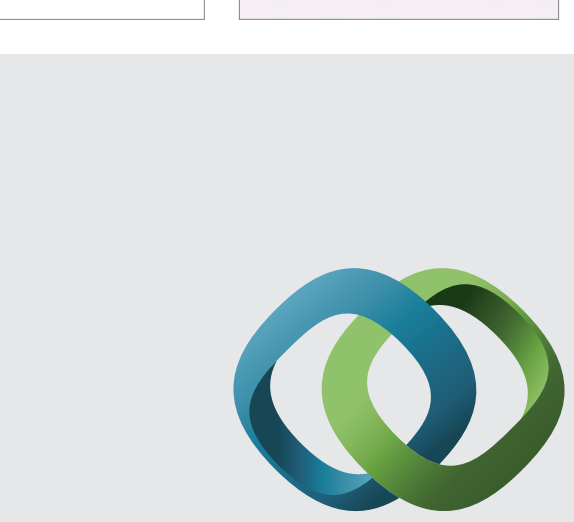

\section{Hindawi}

Submit your manuscripts at

http://www.hindawi.com
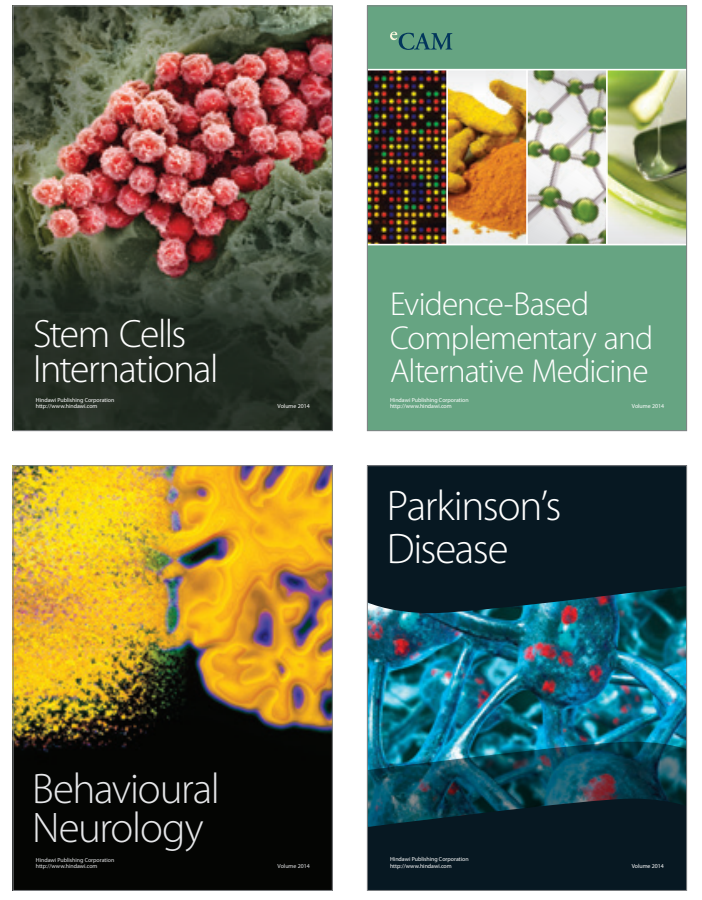
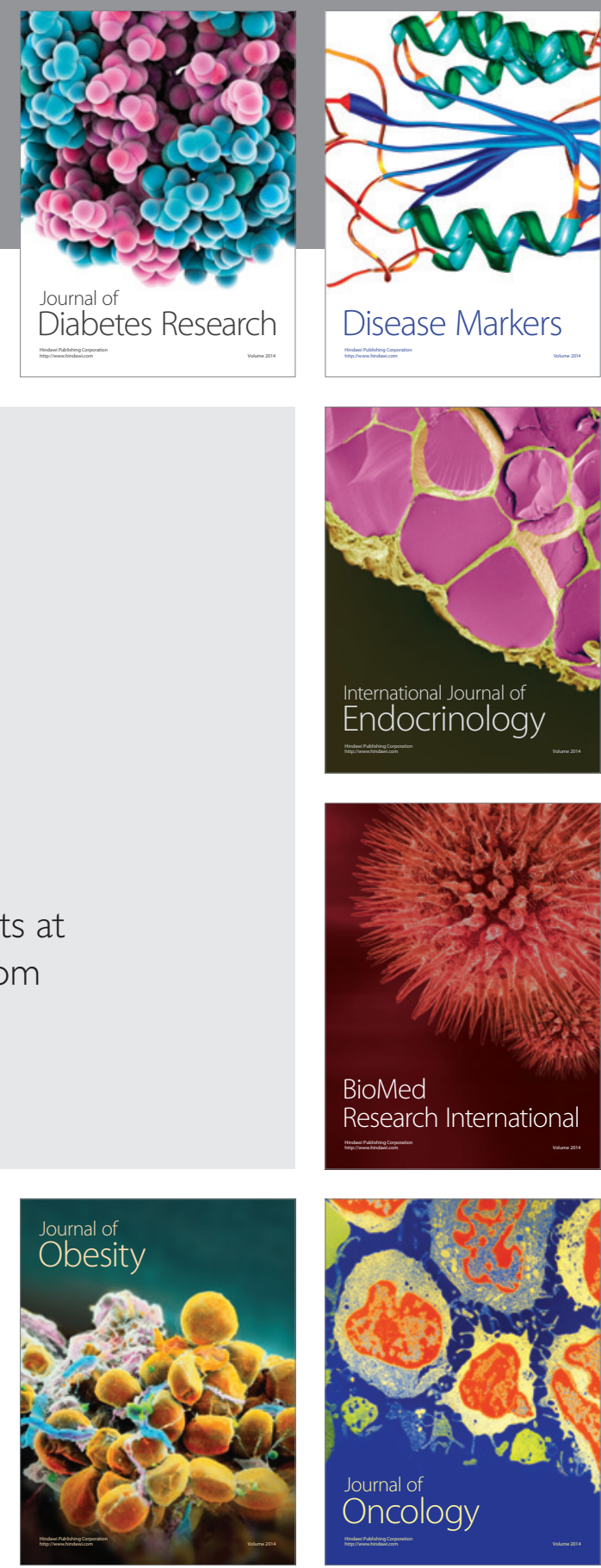

Disease Markers
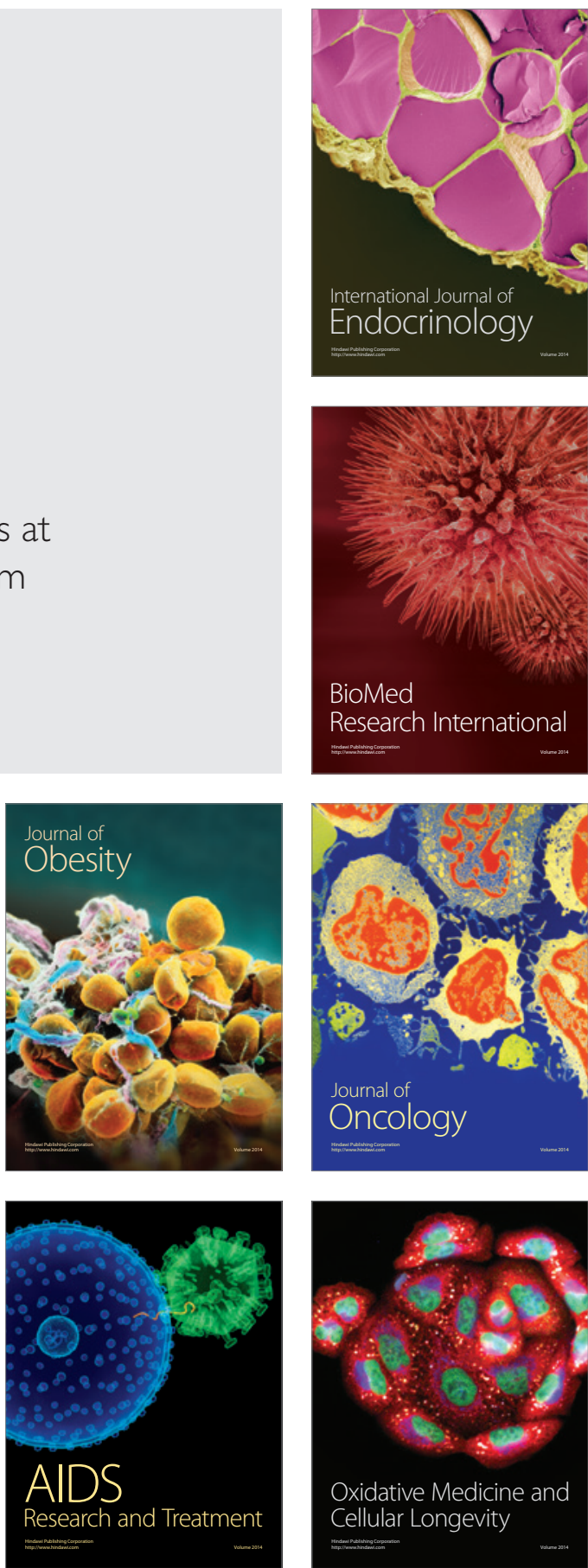